\title{
Arbitrary Unit of Substance Rate
}

National Cancer Institute

\section{Source}

National Cancer Institute. Arbitrary Unit of Substance Rate. NCI Thesaurus. Code C67384.

A arbitrary unit of measurement of substance that passes through or onto a given area per unit of time. 